\title{
5 Bauen wider den Plan
}

Bisher wurde vor allem die reguläre Seite des individuellen Bauwesens in Samarkand besprochen. In der Stadt wurden allerdings auch im großen Umfang eigenmächtig, also ohne Genehmigung, individuelle Häuser gebaut. Das Phänomen war in der gesamten Sowjetunion verbreitet, ${ }^{258}$ wurde mit wechselnder Intensität und ebensolchem Erfolg bekämpft und hat, wie im Falle von Samarkand sichtbar, deutliche Spuren in der Stadtlandschaft hinterlassen.

\section{Samovol'noe stroitel'stvo - die „eigenmächtige Bautätigkeit“"}

Zuerst eine Bemerkung zur Begriffswahl. Bei dieser Form des Wohnungsbaus handelte es sich de facto um illegales bzw. semi-legales Bauen auf einem angeeigneten Stück Land. In den aufgefundenen Dokumenten und Gesetzen wird die Bezeichnung „,illegal“ (nelegal'no) allerdings nicht verwendet, manchmal taucht „gesetzeswidrig“ (nezakonnyi) auf; der fest etablierte und am meisten benutzte Begriff war „eigenmächtige Bautätigkeit“ (samovol'noe stroitel'stvo), den ich in meinen Ausführungen übernehme.

Grundsätzlich kann man den unerlaubten Hausbau in zwei Kategorien unterteilen: einerseits das eigenmächtige Bauen auf individuell angeeigneten Flächen in der Stadt und andererseits das Bauen von Häusern auf dem Land der umliegenden Kolchosen, das durch deren Führung trotz vehementen Widerspruchs der Stadt genehmigt wurde. Genau diese Kategorisierung wurde durch die städtischen Behörden von Samarkand vorgenommen. In der Regel werden in den Dokumenten beide Formen zusammen behandelt. Mir erscheint die Unterteilung dennoch sinnvoll, um beiden Aspekten genügend Raum zu geben. Die zweite Kategorie und den Konflikt, der durch diese Praxis mehrere Jahre lang die Beziehungen zwischen Stadt und Kolchosen prägte, werde ich im nachfolgenden Unterkapitel beschreiben und den Einfluss des Phänomens auf die Stadtstruktur aufzeigen.

In den Archivdokumenten des Samarkander Ispolkom (zum Zeitraum der 1950er- und 60er-Jahre) taucht das Thema gleich zu Beginn der 1950er-Jahre auf. Die Anordnung des Ministerrates der UzSSR Nr. 1695 aus dem Jahr 1951 trägt den

258 Andrusz 1984, S. 99, 106.

Ә Open Access. (C 2021 Marya Petrova, publiziert von De Gruyter. (c) )BY-NC-ND Dieses Werk ist lizenziert unter der Creative Commons Attribution-NonCommercial-NoDerivatives 4.0 International Lizenz. https://doi.org/10.1515/9783110669367-005 
langen Titel „Über die Fakten des Verstoßes gegen den Erlass des SovNarKom der UzSSR vom 16. Dezember 1940 ,Über die Maßnahmen zur Bekämpfung der eigenmächtigen Bautätigkeit in den Städten, Arbeitssiedlungen und Gebietszentren““ und schildert folgende Praxis:

[...] häufig wenden sich Bürger, die eigenmächtig Häuser gebaut haben, an Volksgerichte mit Klagen auf Anerkennung des Rechts auf persönliches Eigentum für die von ihnen errichteten Häuser. Dabei weisen sie Bescheinigungen von städtischen Kommunalwirtschaften und sogar von Büros für Technische Veränderungen (Byuro Tekhnicheskikh Izmenenij BTI) nach, die bestätigen, dass das Haus tatsächlich vom Kläger errichtet worden ist oder dass es ihm gehört und auf eine bestimmte Summe geschätzt wurde. Diese Bescheinigungen erteilen die Organe der Kommunalwirtschaft und des BTI ihrerseits auf Grundlage von Bescheinigungen der Mahalla- bzw. Quartalskomitees.

Auf Grundlage dieser Bescheinigungen erkennen Volksgerichte die Eigentumsansprüche an und unternehmen nichts gegen die eigenmächtigen Handlungen, was bei staatlichen Baumaßnahmen dazu führt, dass Entschädigungen gezahlt werden müssen, womit dem Staat Schaden entsteht. ${ }^{259}$

Das Dokument macht eine Gesetzeslücke deutlich, die sich Bürger zunutze machen konnten, und nennt vier Fälle aus Samarkand mit konkreten Personennamen als Beispiele. Nach der Schilderung der Fälle wird das Ausstellen der entsprechenden Bescheinigungen durch die Organe der Kommunalwirtschaft ausdrücklich verboten und dem Justizministerium wird empfohlen, die Volksgerichte entsprechend anzuweisen. Doch damit war das Problem noch lange nicht aus der Welt, vielmehr wird hier das Phänomen zum ersten Mal in Samarkand belegbar. Auf Allunionsebene wird das Phänomen erst ab Mitte der 1950er-Jahre als Problem wahrgenommen - zumindest lässt sich der im Jahr 1954 zum ersten Mal seit 1940 novellierte Erlass „Über die Bekämpfung der eigenmächtigen Bautätigkeit“ dahingehend deuten. ${ }^{260}$

Die nächste Erwähnung von eigenmächtiger Bautätigkeit konnte ich im Archiv von Samarkand für das Jahr 1956 finden. Der Gebiets-Ispolkom zählt zu dieser Zeit in der Stadt 212 eigenmächtig gebaute Häuser. Als Ursache für diese Fülle wird eine „zu liberale“ Einstellung der lokalen Verwaltung gegenüber den eigenmächtigen Bauherren genannt. Beklagt werden in dem Dokument vor allem die

259 SamOGA, f. 26, op. 1, d. 1406, 1. 15.

260 Erlass des SNK vom 22.05.1940. Die Novellierung bestand in der Streichung eines Punktes zur Registrierung, also dem propiska-Recht (das die Bevölkerungsmobilität kontrollieren und den Zuzug in die Städte regeln sollte), und einer Ermahnung, systematische Kontrollen und den Kampf gegen die eigenmächtige Bautätigkeit ,auf allen Ebenen“ fortzusetzen. 
entgangenen Steuereinnahmen; weiters wird angeordnet, die Häuser schnellstmöglich zu registrieren und zu schätzen, um die Besitzer besteuern zu können. Weitere strafende Maßnahmen werden nicht gefordert. ${ }^{261}$ Zwei Faktoren können diese recht liberale Einstellung erklären: Erstens erlaubte die geltende Rechtslage die Legalisierung von eigenmächtig gebauten Häusern, wenn von ihnen keine Gefahr für Leib und Leben ausging und wenn sie nicht gegen die staatlichen Bebauungspläne verstießen. Zweitens erscheint die Toleranz gegenüber Bauinitiativen seitens der Bevölkerung durch den Wohnungsnotstand, der nach dem Krieg herrschte, nachvollziehbar. Mark Smith betont in diesem Kontext, dass der auf den Zweiten Weltkrieg folgende Wiederaufbau den Bestand an privatem Wohneigentum in der UdSSR vergrößerte und gleichzeitig die politische Toleranz gegenüber dem Phänomen erhöhte, da viele Menschen dies als einen Ausgleich oder als verdiente Belohnung nach den Leiden und Strapazen des Krieges betrachteten. ${ }^{262}$ Dass es sich im Falle von Samarkand bei dieser Art von Häusern um sehr einfache und kleine Bauten handelte, wurde oben bereits angesprochen.

Die Rhetorik verschärft sich allerdings im November desselben Jahres, und auch die Zahl der Meldungen über unerlaubte Bauten steigt: In den beiden Kategorien (d.h. sowohl auf Stadt- als auch auf Kolchos-Land gebaut) werden zusammen über 600 Häuser gezählt. ${ }^{263}$ Viele dieser Bauten wurden dem Bericht zufolge in den „Flussbetten der Bewässerungskanäle“ gebaut. Gemeint sind für das Grund- und Stadtrelief von Samarkand spezifische, durch alte Bewässerungskanäle und kleine Flüsse gebildete Schluchten bzw. Erosionsgräben (russ. ovragi). Sie sind abseits der zentralen Straßen und Plätze der Stadt gelegen und boten damals offenbar genügend Blickschutz; ihr schlechter, instabiler Boden macht sie aber nicht besonders geeignet für den Hausbau. Auch auf Flächen, die eigentlich für planmäßige Bebauung vorgesehen waren, wurden unrechtmäßig gebaute Häuser registriert. Von den zuständigen Behörden einschließlich der Polizei werden schärfere Kontrollen und härteres Durchgreifen gefordert. Gleichzeitig wird für Häuser, die vom Abriss verschont bleiben sollten, eine Registrierung zum Zwecke der Besteuerung angeordnet. Zusätzlich wird allerdings darauf hingewiesen, dass eine Steuerzahlung noch kein Recht auf „unbefristete Nutzung“ (bessrochnoe pol'zovanie) mit sich bringe. ${ }^{264}$

261 SamOGA, f. 1617, op. 1, d. 62, 1. 75-76.

262 Smith 2010, S. 154-155.

263 SamOGA, f. 1617, op. 1, d. 62, 1. 79-80.

264 SamOGA, f. 1617, op. 1, d. 62, 1. 79-80 
Vier Jahre später, im Jahr 1960, enthielt der Bericht der Wohnraumbestandszählung noch höhere Zahlen: Demnach wurden in Samarkand mindestens 2.600 nicht registrierte, eigenmächtig errichtete Bauten entdeckt, von denen die städtischen Behörden davor nicht informiert waren. ${ }^{265}$ Vermutlich handelt es sich bei dieser Zahl erneut um beide Kategorien, also Hausbau auf Kolchos-Land eingeschlossen. Es bleibt leider unklar, ob es sich hier um einen tatsächlichen Anstieg oder nur um eine genauere Zählung handelt. Es sei jedoch in Erinnerung gerufen, dass sich parallel der Bauboom von 1957-1959 ereignete (s. Kapitel 4). Es ist möglich, dass es im Schatten einer großen Kampagne für individuellen Wohnungsbau leichter war, ein Grundstück an sich zu ziehen. Insgesamt war das Problem der unregistrierten, eigenmächtig gebauten Häuser in zahlreichen Städten der Republik verbreitet, allen voran in Taschkent mit 34.000 (!) Häusern. ${ }^{266}$

Im Jahr 1961 bezeichnet ein Bericht zum Stand der Industrie- und Wohnungsbaumaßnahmen in Samarkand die eigenmächtige Bautätigkeit als das „größte Übel für die richtige Bebauung der Städte und ländlichen Siedlungen“. Hier wird bereits nicht mehr die Zahl der Häuser, sondern nur mehr die ungefähre derart verbaute Fläche genannt, die auf recht beeindruckende 350 Hektar geschätzt wurde. ${ }^{267}$ Dies hätte ca. 4.500 planmäßig angelegten Standardparzellen entsprochen $^{268}$, womit deutlich wird, dass der Umfang der eigenmächtigen Bebauung etwa dem der planmäßigen glich, wenn er diese nicht sogar überstieg. (In den Jahren des Baubooms zwischen 1957 und 1959 wurden 250 Hektar planmäßig bebaut. ${ }^{269}$ ) Dieses Ausmaß an unkontrollierter Bebauung stellte die Stadt vor große Schwierigkeiten, da dadurch zahlreiche Flächen belegt wurden, die für andere Vorhaben eingeplant waren, sodass diese Bauprojekte verhindert wurden; genau darüber klagt der Bericht. Die größte Schuld wird dabei den Kolchos-Leitern zugeschrieben, aber gleichzeitig werden sowohl die Leitung des Stadt- und des Gebiets-Ispolkom als auch die Parteiführung beschuldigt - die einen wegen unzureichender Kontrolle und die anderen wegen ,unzureichender Aufmerksamkeit“ dem Problem gegenüber. ${ }^{270}$

265 TsGARUz, f. 1619, op. 16, d. 4, 1. 25-26.

266 TsGARUz, f. 1619, op. 16, d. 4, 1. 23-24.

267 SamOGA, f. 1617, op. 1, d. 126, 1. 52.

268 Berechnet auf der Grundlage einer Aufstellung aus dem Jahr 1959, siehe SamOGA, f. 26, op. 1. d. 2268, 1. 50. Darin wird der Bedarf der Stadt an Landflächen vorgerechnet, wonach für 1530 Häuser mit einer Parzellengröße von $450 \mathrm{~m}^{2}$ insgesamt 110 Hektar Land benötigt wird, Flächen für Straßen und öffentliche Infrastruktur eingerechnet.

269 SamOGA, f. 26, op. 1, d. 2168, 1. 322.

270 SamOGA, f. 1617, op. 1, d. 65, 1. 102. 
Unerlaubt gebaute Häuser sollten nach entsprechender Aufforderung des Ispolkom innerhalb eines Monats auf eigene Kosten zurückgebaut bzw. abgerissen werden. Dies war der Mindestkonsens; ansonsten unterschieden sich die Strafmaßnahmen in ihrer Strenge offenbar je nach Unionsrepublik: Während es im Strafgesetzbuch der RSFSR einen eigenen Artikel dafür gab, ${ }^{271}$ fehlt ein vergleichbarer Eintrag im Strafgesetzbuch der Usbekischen SSR gänzlich. ${ }^{272}$ In der Usbekischen Republik war es ein Beschluss des Präsidiums des Obersten Sowjets von 1960 (der bis in die 1970er in Geltung blieb), der die Angelegenheiten rund um die eigenmächtige Bautätigkeit regelte. Neben dem Abriss des unberechtigt Gebauten sah dieser Beschluss eine Geldstrafe in Höhe von 3000 Rubel vor. ${ }^{273}$ Das Strafgesetzbuch der RSFSR aus dem Jahr 1962 enthält zwar eine geringere Geldstrafe (100 Rubel, entspricht 1000 Rubel vor der Währungsreform von 1961), dafür droht es aber eine weitere Strafmaßnahme an, nämlich Strafarbeiten (ispravitel'nye raboty) für die Dauer von sechs Monaten bis zu einem Jahr. Gleichzeitig existierte die bereits beschriebene Möglichkeit der rückwirkenden Legalisierung. Bemerkenswert ist, dass im Jahr 1962 das Plenum des Obersten Gerichts anordnete, dass die Zuständigkeit, die entsprechenden Entscheidungen zu treffen, allein beim örtlichen Ispolkom und nicht bei den Gerichten liegen sollte. ${ }^{274}$ Oksana Gumilevskaya schreibt, dass in der RSFSR strenge Sanktionen nur selten angewendet wurden; ${ }^{275}$ eine ähnliche Situation zeigt sich auch in Samarkand.

Die Archivmaterialien der Samarkander Behörden enthalten zahlreiche Zeugnisse dafür, dass es der Stadtverwaltung nicht gelang, die privaten Bauvorgänge in der Stadt zu kontrollieren. Am anschaulichsten wird das am Schriftwechsel zwischen den diversen Verantwortungsebenen der Republik deutlich. Der Ispolkom von Samarkand wird immer wieder für die schlechte Kontrolle über Grund und Boden kritisiert, aber auch für andere Verstöße im individuellen Wohnungsbau, darunter illegale Grundstücksverkäufe und Schenkungen, die zulässigen Normen übersteigender Hausbau sowie der Anbau von Obst und Gemüse zum illegalen Vertrieb, betrieben auf eigenmächtig angeeigneten Flächen. ${ }^{276}$ Nicht ganz nachvollziehbar wirkt in diesem Kontext der Umstand, dass trotz der kritischen Situation die Anzahl der Mitarbeiter der Inspektionsorgane verkleinert

271 Strafgesetzbuch der RSFSR von 1960, Art. 199.

272 Strafgesetzbuch der UzSSR von 1960.

273 Vedomosti Verkhovnogo Soveta UzSSR 1960 Nr. 26 S. 31-32.

274 Byulleten' Verkhovnogo Suda SSSR 1962, S. 24, Punkt 10.

275 Gumilevskaya 2008, S. 98. 1963 wurde die lockere Rechtspraxis in einem Beschluss des Plenums des Obersten Gerichts der UdSSR kritisiert.

276 SamOGA, f. 1617, op. 1, d. 118, 1. 14. 
wurde. ${ }^{277}$ Scharf kritisiert wird die Stadtverwaltung auch für ihr zu spätes Eingreifen, nämlich in der Regel erst wenn die Häuser bereits standen, für zu wenig Entscheide auf Strafen und Abriss, für die Unfähigkeit, solche am Ende auch zu vollstrecken, sowie dafür, dass die verantwortlichen Personen straffrei ausgingen. ${ }^{278}$

Die Vorwürfe kommen sowohl von der Ebene des Gebiets-Ispolkom als auch vom Ministerrat der UzSSR. Der Ispolkom von Samarkand seinerseits gab die Vorwürfe häufig nach „unten“ an die Architektur- und Baubehörde weiter, in deren Aufgabenbereich auch die Kontrolle über die Bauvorgänge in der Stadt gehörte, bzw. an die Exkutivkomitees der Bezirke, durch deren milde Entscheidungen oder unterstellte Nachlässigkeit bei der Ahndung der eigenmächtige Bau in diesem Ausmaß erst möglich geworden war. ${ }^{279}$ Nach „oben“ reichte der Stadt-Ispolkom Berichte über die aufgedeckten Fälle von unerlaubt gebauten Häusern und über die verhängten Strafen weiter, jedoch sind die Zahlen nicht besonders hoch. So wird etwa im Jahr 1961 von dreißig auferlegten Geldstrafen berichtet ${ }^{280}$ und im Jahr 1963 von vierzig aufgedeckten Fällen, die mit Strafen belegt wurden. Wie viel davon tatsächlich umgesetzt wurde, ist schwer zu sagen - sicher ist, dass es einige Abrisse gab, sicherlich auch zur Abschreckung. Ein Gesprächspartner, der Ende der 1960er-Jahre in einer Baubehörde arbeitete, deutete an, dass solche Aktionen zwar stattfanden, aber eher Exempel-Charakter hatten. ${ }^{281}$

Die Frage, die sich dabei stellt, ist die nach dem Vorhandensein einer gewissen Hemmschwelle bei staatlichen Behörden bzw. Entscheidungsträgern in einem sozialistischen Staat, Menschen auf die Straße zu setzen, denn dies wäre die reelle Folge eines Abrisses gewesen. In den Dokumenten wird die Frage, was mit den Menschen passiert, nicht aufgeworfen. Offenbar war hier der Staat zwischen zwei seiner Aufgaben - der sozialen Versorgung und der Kontrolle über die Einhaltung der Regeln - gefangen. ${ }^{282}$ Die aufgefundenen Beispiele für den Umgang der Exekutivkomitees mit den Anträgen auf Legalisierung unerlaubt gebauter Häuser lassen keine konsequente Haltung nachvollziehen. Genauso wenig geben sie Einblick in die Beweggründe hinter der einen oder der anderen Entscheidung. Die abgewiesenen Anträge auf Legalisierung ließen die Antragsteller offenbar in einer rechtlichen Grauzone zurück. So wird z.B. in einem Fall der Antrag beim Stadt-Ispolkom auf Registrierung des Hauses zwar abgelehnt, der Person wird

277 SamOGA, f. 1658, op. 2, d. 109, 1. 201-202.

278 SamOGA, f. 1617, op. 1, d. 122, 1. 47.

279 SamOGA, f. 1617, op. 1, d. 83, 1. 61.

280 SamOGA, f. 1658, op. 2, d. 109, 1. 201.

281 Gespäch mit I.B.

282 Für diese Beobachtung und den Hinweis bedanke ich mich bei Udo Grashoff. 
aber empfohlen, sich an das Büro für Technische Inventarisierung (BTI) zu wenden, um darüber das Eigentumsrecht anerkannt zu bekommen, ${ }^{283}$ also genau die Praxis, die am Eingang dieses Kapitels beschrieben wurde und die theoretisch verboten war.

Ein anderes Beispiel berichtet von sechs Bürgern, die - angeblich wissentlich - eigenmächtig Häuser auf Grundstücken unter geplanten Hochspannungsleitungen errichteten; sie erhielten als Ersatz neue Parzellen. ${ }^{284}$ Das bedeutet, dass die Behörden die Häuser aus Sicherheitsgründen abreißen mussten und dabei auch noch gezwungen waren, Ersatzgrundstücke zuzuteilen. Um solche paradoxen Situationen zwischen den Aufgaben der Behörden und den Bedürfnissen der Bürger zu verstehen, ist die Analyse von Mark Smith hilfreich. Die Handlungen von Menschen, die eigenständig und zuweilen auch eigenmächtig ihre Häuser gebaut hatten, bezeichnet er als „sanctioned initiative“ und „permitted autonomy“. Gemeint ist damit, dass die herrschende Wohnungsnot den Staat dazu zwang, die Eigenmächtigkeit seiner Bürger zu tolerieren, ja sich geradezu auf diese $\mathrm{zu}$ verlassen, weil die staatlichen Ressourcen allein nicht zur Beseitigung der Wohnungsnot ausreichten. Smith betont an der Stelle, dass die Versuche, die Wohnungsnot eigenhändig zu lösen und damit nicht immer im Einklang mit geltendem Recht zu handeln, nicht als Widerstand gegen das System, sondern vielmehr in den Kategorien von Kooperation oder Symbiose beschrieben werden können.

Diese Perspektive hilft bei der Beantwortung der Frage, warum der eigenmächtige Hausbau solche Ausmaße annehmen und so schlecht bekämpft werden konnte. Ein Teil der aufschlussreichen Argumentation von Smith ist die These, dass die Eigeninitiative der Bürger de facto Teil des Systems war und dessen Funktionieren erst ermöglichte. ${ }^{285}$ Diese Beobachtung lässt aber die Frage offen, inwiefern die beteiligten Akteure, vor allem diejenigen auf der Seite des Staates, sich der „Symbiose“ bewusst waren und aus welchen Motivationen und Handlungen der Verantwortlichen in der Verwaltung diese „Kooperation“ entstand.

Die Überforderung der Behörden und der einzelnen Mitarbeiter, ihre ambivalente Position in der Entscheidungshierarchie, aber auch ihre eigenen Interessen und Vorteile spielen hier ebenso eine wesentliche Rolle. Vor allem gilt dies für die Architektur- und Baubehörde, da diese neben der Kontrolle zugleich für die Durchsetzung der Strafen zuständig war. Zur Überforderung der Behörden trug

283 SamOGA, f. 1658, op. 2, d. 109, 1. 51.

284 SamOGA, f. 1658, op. 2, d. 109, 1. 222.

285 Smith 2010, S. 14-15, 140-141. 
die offenbar häufig nicht geklärte Zuständigkeit bei, die durch Mehrfachkompetenzen entstanden war. Die Bezirks-Ispolkom genehmigten zum Beispiel häufig die Zuteilung von Grundstücken, wo die städtische Architekturbehörde vorher die Zuteilung untersagt hatte. Ebenso konnte es geschehen, dass Mitarbeiter der Architekturbehörde, die nicht dazu befugt waren, Genehmigungen ausstellten, die später nicht mehr rückgängig zu machen waren. Dadurch entstanden Verstöße gegen den Bebauungsplan. Die Verantwortung dafür wird in den Archivquellen vor allem der Architekturbehörde zugeschrieben, die all diese Probleme zwar bemerkte und kritisierte, sie aber letztlich nicht effektiv verhindern konnte. ${ }^{286}$ Offenbar herrschte Unklarheit darüber, wie mit fehlerhaften Entscheidungen, die bereits Tatsachen geschaffen hatten, umgegangen werden sollte. Ein weiterer nicht zu unterschätzender Faktor bei den Aushandlungsprozessen waren sicherlich persönliche Interessen der Bearbeiter, sei es durch Korruption, als „Gefälligkeiten“ oder aus Verständnis für die Situation der Bittsteller. Natürlich sind solche Praktiken nur schwer in den Quellen belegbar, auch wenn sie in vielen Situationen implizit mitgedacht werden müssen.

Die geschilderte Situation stellt keinesfalls eine Besonderheit Samarkands dar. Mehrere Autoren, darunter Thomas Bohn, Gregory Andrusz und auch Mark Smith, erwähnen ähnliche Situationen für andere Teile der Sowjetunion. ${ }^{287}$

\section{Bodenkonflikte zwischen Stadt und Kolchosen}

In den bisherigen Ausführungen wurde bereits häufig genug auf diesen Abschnitt verwiesen, der als letzter Mosaikstein bei der Beantwortung der Frage nach den Gründen für die Entstehung der Stadtstruktur von Samarkand dienen soll. Der Bodenkonflikt zwischen der Stadt und den umliegenden Kolchosen, um den es hier gehen soll, hielt über mehrere Jahre an und hatte einen sehr prägenden Einfluss auf die Stadtplanungsprozesse und damit auf das heutige Stadtbild von Samarkand. Diesen Einfluss werde ich versuchen zu umreißen. Gleichzeitig werde ich auf der Grundlage der zur Verfügung stehenden Dokumente vor allem die Perspektive des Stadt- und Gebiets-Ispolkom und der zugehörigen Bau- und Architekturbehörden wiedergeben; die Perspektive der Kolchosen spiegelt sich in den Quellen nur in sehr geringem Maße.

286 SamOGA, f. 26, op. 1, d. 1675, 1. 54-55.

287 Andrusz 1984, S. 102-103; Smith 2010, S. 15; Bohn 2008, S. 176 ff. 
Der Konflikt war gewissermaßen vorprogrammiert, da in der Usbekischen Sowjetrepublik das Oasenland, das sich Städte und Landwirtschaft teilen mussten, nur 6\% der gesamten Fläche ausmachte. ${ }^{288}$ Der Anfang der intensiven Phase lässt sich für das Jahr 1956 feststellen, als mit der Fertigstellung des neuen Generalplans und seiner Genehmigung durch den Ministerrat der UzSSR auch „perspektivisches Bauland“ für Samarkand im Umfang von insgesamt ca. 1.500 Hektar ausgewiesen wurde, das bis dahin zu den Territorien der umliegenden Kolchosen gehörte. ${ }^{289}$ Damit sollten Territorien, die unmittelbar an Samarkand grenzten, von den Kolchosen an die Stadt übergeben werden. Das formelle Prozedere sah eine Stellungnahme seitens der Kolchosversammlung vor. ${ }^{290}$ Inwiefern dabei eine ausdrückliche Zustimmung erforderlich war und ob diese eine reine Formalität darstellte oder die Versammlung doch ein tatsächliches Entscheidungsinstrument der Kolchosen war, ist sicher eine wichtige Frage, um den Mechanismus dieses Konfliktes nachzuvollziehen, sie wird sich aber im Rahmen dieser Studie leider nicht eindeutig beantworten lassen. Klar ist nur, dass zahlreiche Kolchosen von der Möglichkeit aktiv Gebrauch machten und sich noch mit unterschiedlichen anderen ihnen zugänglichen Mitteln gegen die Landübereignung zu wehren versuchten. In den Archivdokumenten findet man Berichte, die von unterschiedlichen Taktiken zeugen, vom Ignorieren der Beschlüsse über die Verschleppung von bürokratischen Abläufen bis hin zu einer direkten Absage oder Verhinderung von Baumaßnahmen. ${ }^{291}$

Das größte Problem für die Stadt und die Architektur- und Baubehörde war an dieser Situation, dass die Kolchosen zusätzlich zu der genannten Verweigerungshaltung auch praktisch die umstrittenen Territorien mit individuellen Häusern zubauen ließen. Bei einigen Kolchosen reichten die Grundstücke weit in die zentralen Bereiche der Stadt hinein, und so waren der Stadt-Ispolkom und seine Behörden auf einmal mit unkontrollierter Bebauung von wertvollem Land kon-

288 Bulatov 1962, S. 11.

289 SamOGA, f. 26, op. 1, d. 1932, 1. 3-4.

290 Beschluss des Ministerrates der RSFSR vom 13.09.1954 (in: Sbornik zakonodatel'nykh aktov o zemle 1960, S. 45). In der Liste der Dokumente, welche mit dem Antrag auf Landübereignung beim Ministerrat der Unionsrepublik eingereicht werden sollten, wird auch ein „Protokoll der beschlussfähigen Versammlung der Kolchosmitglieder“ aufgelistet. Die Rede ist dabei nicht von einer Entscheidung der Versammlung - diese sollte vom Bezirks-Ispolkom kommen. Welches Gewicht die Meinung der Kolchosmitglieder dabei hatte, wird nicht erwähnt. Da mir der entsprechende Beschluss der UzSSR nicht vorgelegen hat, bin ich gezwungen, mich auf den der RSFSR $\mathrm{zu}$ beziehen.

291 SamOGA, f. 26, op. 1, d. 1932, 1. 20. 
frontiert, welche zahlreiche städtebauliche Vorhaben nichtig und den Generalplan unumsetzbar machte. Von der Stadt wurde dies als eigenmächtige Bautätigkeit deklariert; allerdings hatten die Behörden hier noch weniger Handlungsmöglichkeiten, da der individuelle Häuserbau in diesen Fällen durch die Genehmigungen seitens der Kolchosen rechtlich abgesichert war. In zahlreichen Beschwerde- und Drohbriefen wird eine Situation beschrieben, bei der die Parzellen in großem Maße auch an Nicht-Kolchosmitglieder verteilt und Grundstücke völlig ohne Plan und Infrastruktur bebaut wurden. ${ }^{292}$

Samarkand stellt auch hier wieder keinen Einzelfall dar: Dem Erlass des Ministerrates der UzSSR vom April 1957 zufolge waren solche Fälle ein Problem in der gesamten Republik. ${ }^{293}$ In den Dokumenten zu Samarkand tauchen die Namen von insgesamt zehn bis zwölf Kolchosen auf, die die Stadt umgaben.

\section{Der Fall „Kaganovich“-Kolchos}

Am besten überliefert ist der Konflikt mit der Leitung des „Kaganovich“-Kolchos Mitte bis Ende der 1950er-Jahre. In der Auseinandersetzung mit der Stadt holte sich dieser Kolchos offenbar Rückendeckung seitens des Landwirtschaftsministeriums, sodass der Konflikt letztlich auf eine höhere Ebene überging. Hinweise darauf enthält ein Erklärungsschreiben des stellvertretenden Vorsitzenden des Stadt-Ispolkom, Akulyshin, an den Ministerrat der UzSSR vom August 1956. ${ }^{294}$ Darin kritisiert er, das Landwirtschaftsministerium würde sich in Bezug auf den Konflikt einseitig als Interessensvertretung der Kolchosen positionieren, statt das gesamtstaatliche Interesse zu vertreten. Er akzeptiert zwar einen der Hauptkritikpunkte, nämlich den Wegfall von fruchtbaren Böden und die damit einhergehenden Nachteile für die Produktionskraft der Kolchosen, rechtfertigt das Handeln der Kolchosen aber mit dem Hinweis, dass es um Samarkand herum ,keine anderen Territorien [gäbe], die nicht Obst- oder Weingärten wären““. ${ }^{295}$ Er warnt davor, dass die Priorisierung der Kolchosinteressen eine Revision und Neuausrichtung des Generalplans von Samarkand, der bereits vom Ministerrat der Republik genehmigt worden war, bedeuten würde. Letztlich fordert er eine unabhängige

292 Von dieser Praxis, an Parzellen heranzukommen, erzählten mir auch zwei Gesprächspartner, die zu Sowjetzeiten als Architekten bzw. Mitarbeiter der Architekturbehörde tätig waren (Gespräch mit B.R. und I.B.).

293 SamOGA, f. 1617, op. 1, d. 82, 1. 1.

294 SamOGA, f. 26, op. 1, d. 1932, 1. 3-7.

295 SamOGA, f. 26, op. 1, d. 1932, 1. 3-7. 
Kommission, welche die einander widersprechenden Angaben der Konfliktparteien überprüfen solle. Ob tatsächlich eine Kommission eingesetzt wurde und ob Akulyshins Kommentare und Forderungen berücksichtigt wurden, lässt sich anhand der zur Verfügung stehenden Quellen leider nicht nachvollziehen; es wird aber deutlich, dass das Problem auf unterschiedlichen Ebenen verhandelt wurde. Der Ministerrat reagierte im April 1957 auf die Problemlage mit einem Beschluss, ${ }^{296}$ der die Gründe für die „,schädliche Praxis“ nennt:

[..] Defizite in der Arbeit des staatlichen Komitees für Bau und Architektur des Ministerrates der UzSSR sowie der Projektorganisationen, die bei der Erarbeitung von Generalplänen der Städte die Fragen einer rationalen Bodennutzung durch die Kolchosen sowie die der Platzierung von Bauobjekten von Kolchosen auf in die Stadtgrenzen eingegangenen Territorien nicht berücksichtigt haben. ${ }^{297}$

Besonders bemerkenswert ist, dass der Ministerrat von einem ausdrücklichen Verbot der Bebauungspraxis absieht und sich lediglich auf eine sanftere „Empfehlung, keine Bautätigkeit innerhalb der Projektgrenzen der Stadt auszuführen“ beschränkt. Diese Formulierung wird auf unterschiedlichen Ebenen im Wortlaut in zahlreichen Beschlüssen und Erlässen der darauffolgenden Jahre übernommen. Als Ausnahme wird der Bau von leichten temporären Wirtschaftsgebäuden in Abstimmung mit den lokalen Ispolkoms genehmigt. Ansonsten bleiben die Verordnungen sehr allgemein und uneindeutig: Eine bessere Koordinierung und Abstimmung zwischen Ministerien, Planungsbehörden und Kolchosen wird angeordnet; die Vorschläge zum Wohnungsbau in stadtnahen Kolchosen sollten in Abstimmung mit den Generalplänen der Städte erarbeitet werden. Nicht explizit, sondern als Nebensatz eingeschoben - wodurch seine Wirkmächtigkeit nicht ganz klar wird - steht der Hinweis, Kolchosland innerhalb der Stadtgrenzen sei nur für landwirtschaftliche Kulturen zu verwenden.

Der Beschluss macht zwar Vorschläge für die Zukunft, entzieht sich aber hinsichtlich schon bestehender Konflikte einer eindeutigen Aussage oder auch der Verantwortung und leitet diese nach unten weiter. Der Beschluss ist sicherlich das Ergebnis eines internen Aushandlungsprozesses, jedoch konnte der Ministerrat zu dem Zeitpunkt im Konflikt zwischen Bau- und Landwirtschaftsministerium keine eindeutige Entscheidung fällen, was dazu führte, dass der status quo beibehalten wurde.

296 SamOGA, f. 1617, op. 1, d. 82, 1. 1-2. Beschluss des Ministerrates der UzSSR vom 01.04.1957 „Über die Regulierung der Bebauung von Kolchosländereien innerhalb der Grenzen der Städte.“ 297 Übersetzung der Autorin. 
Da der Beschluss bloß eine bessere Planung verordnete, trug er natürlich nicht zur Lösung des Konflikts bei, und so häuften sich weitere Klagen und Beschwerdebriefe. So hieß es im Jahr 1959, dass „die eigenmächtige Baupraxis seitens der Kolchosen in den letzten Jahren Massencharakter“ angenommen habe und „die staatlichen Baupläne zu sprengen“ ${ }^{298}$ drohe.

Auf der anderen Seite lässt sich die Abwehrhaltung der Kolchosen durchaus nachvollziehen. Ein Beispiel aus dem Jahr 1958 zeigt eine Situation, in der sich Kolchosen weigerten, im laufenden Jahr Land an die Stadt abzutreten. In der Beschwerde wendet sich der Leiter der städtischen Baubehörde an das Gebiet-Ispolkom mit der Bitte, dieses möge auf die Kolchosen einwirken, 100 Hektar des geforderten Landes sofort und weitere 100 Hektar nach der Ernte zu übergeben. ${ }^{299}$ Das Beispiel lässt erahnen, dass die Kolchosen vor dem Problem standen, mit ihrer Ernte ein Plansoll erfüllen zu müssen, das natürlich bei Landabtretung vor der Ernte gefährdet gewesen wäre. Einige Berichte geben knapp die Position der Kolchosen wieder: Darin beziehen sie sich vor allem auf „zur zeitlich unbefristeten Nutzung“ übergebenes Land und auf Beschlüsse der Mitgliederversammlung. ${ }^{300}$ In einem anderen Fall sollen die Kolchosleitungen ihren Widerstand damit begründet haben, dass sie nicht in die Planungen des Generalplans einbezogen waren. ${ }^{301}$ Exemplarisch für die Konflikte, die zwischen Stadt und Kolchosen immer wieder entstanden, soll hier noch der Fall des Engels-Kolchos dargelegt werden.

\section{Der Fall „Engels“-Kolchos}

Hier geht es um einen Konflikt der Stadt Samarkand mit dem Kolchos „Engels“, dessen Territorien im Nordwesten in die sogenannten Projektgrenzen der Stadt eingeschlossen worden waren. Dieser Konflikt lässt sich anhand von zwei Berichten $^{302}$ rekonstruieren und zeigt exemplarisch das Spannungsfeld auf, in dem sich Stadtplanung versus Ressourcenpolitik verorten lassen.

298 SamOGA, f. 1617, op. 1, d. 121, 1. 54.

299 SamOGA, f. 1617, op. 1, d. 83, 11. 25-26.

300 SamOGA, f. 26, op. 1, d. 1932, 1. 2.

301 SamOGA, f. 26, op.1, d. 1932, 1. 17.

302 SamOGA, f. 1617, op. 1, d. 126, 11. 20-23 und 35-38. Der erste, verfasst im Mai 1961 vom Leiter der Städtischen Abteilung für Planung und Architektur Abdullaev, ist an den Vorsitzenden des Gebiets-Ispolkom gerichtet. Der zweite, vom Juli desselben Jahres, verfasst von dem Stellvertre- 
Wie auch bei anderen Kolchosen, wurde „Engels“ im Jahr 1956 mit den Ergebnissen des neuen Generalplans für die Gestaltung von Samarkand bekannt gemacht und darüber informiert, welche Territorien nun ins Stadtgebiet eingegangen waren und im Laufe der Baumaßnahmen an die Stadt übereignet werden sollten. Konkret war ein Grundstück für die Errichtung einer Industriezone mit einer Kammgarnspinnerei im nordwestlichen Teil der Stadt eingeplant. Die Kolchosleitung hatte jedoch die betreffenden Territorien bereits als Parzellen zur individuellen Bebauung vergeben. Im April 1958 brachte der Ispolkom von Samarkand eine Veränderung in den Plan hinein und reservierte in Absprache mit dem Kolchos einen Teil des umstrittenen Territoriums für die Vergabe von Parzellen zur individuellen Bebauung; im Mai 1959 nahm die Stadt diese Entscheidung jedoch wieder zurück.

Laut Bericht nahm die Kolchosleitung - und zwar erst nachdem die Generalplanveränderung bereits zurückgezogen worden war - eine besonders intensive Parzellenverteilung vor. Der Leitung und vor allem der Kolchosvorsitzenden Fatima Kasymova werden ähnliche Verstöße auch in anderen Stadtteilen vorgeworfen: Insgesamt sei über 100 Hektar Kolchosland unautorisiert zur individuellen Bebauung freigegeben worden. Weiter wird Kasymova beschuldigt, die Entscheidungen des Gebiets-Ispolkom und sogar des Ministerrates der UzSSR zu ignorieren. Ebenso wird eine Reihe von Briefen, Beschlüssen und Warnungen aufgezählt, auf welche die Kolchosleitung ebenfalls nicht reagiert habe.

Es wird auch festgestellt, dass 11 von insgesamt 37 gebauten Häusern von Individuen errichtet wurden, die eine Position in der Verwaltung von Stadt oder Kolchos innehatten, einschließlich der Vorsitzenden selbst. Der Bericht wagt allerdings keine Schlüsse in Richtung Begünstigung, Korruption o.ä. zu ziehen; es bleibt bei der Nennung der Namen sowie Hinweisen darauf, dass einige der Häuser die zulässige Größe überschritten.

Letztlich fand eine Regierungskommission im Jahr 1960 eine Kompromisslösung für die Platzierung der Spinnerei, sodass nur zwölf Häuser, die bis dahin auf dem strittigen Land errichtet worden waren, abgerissen werden sollten. Die Kompromisslösung wurde mit der Vermeidung höherer Kosten begründet, die im Falle eines kompletten Abrisses entstünden. Die Besitzer der abgerissenen Häuser sollten dem Bericht zufolge Ersatzgrundstücke, Darlehen, Baumaterialien und sogar Arbeitshilfe beim Wiederaufbau bekommen - kompensatorische Maßnahmen, die zusammengenommen betrachtet eher wie eine Sonderbehandlung

tendem Vorsitzenden des Samarkander Gebiets-Ispolkom Kuzmin, ist an den Sekretär des Parteikomitees des Gebiets von Samarkand adressiert. Der Fall wird damit von „unten“ nach „oben“ weitergereicht. 
wirken. Bezeichnend ist auch, dass der zweite Bericht an die Parteiführung leicht abgemildert formuliert ist. Er enthält zwar die Beschreibung der Kompromisslösung und der Kompensationen, lässt aber nunmehr die Liste mit den Namen der Kolchosfunktionäre weg.

Worauf sich die selbstbewusste Widerspenstigkeit der Kolchosleitung gründete, wird verständlicher, wenn wir den Kolchos sowie die Rolle und Persönlichkeit der Vorsitzenden in größerem Kontext anschauen. Ein Filmbeitrag aus dem Jahr 1968 porträtiert Fatima Kasymova - zu dem Zeitpunkt seit 15 Jahren gewählte Vorsitzende des Kolchos - als eine starke und selbstbewusste Frau an der Spitze eines großen und reichen Kolchos mit mehreren Wirtschaftszweigen. ${ }^{303}$ In seinem landwirtschaftlichen Betrieb werden die verschiedensten Obst- und Gemüsesorten angebaut sowie Schaumwein produziert. Ich konnte nicht herausfinden, ob der Kolchos zu den sog. Millionär-Kolchosen gehörte, aber offenbar war er wirtschaftlich nicht unbedeutend - z.B. als wichtiger Lebensmittelzulieferer. Vorsitzende Kasymova war ihrerseits, wie viele Kolchos-Leiter, Abgeordnete des Obersten Sowjets der Republik und besaß einigen Quellen zufolge den Orden „Held der Sozialistischen Arbeit“. Zudem verfügte sie über hohen moralischen Kredit, indem sie mit ihrem Mann zu den angesehenen Familien in der Republik zählte, die während des Zweiten Weltkriegs mehrere Kinder adoptierten; ${ }^{304}$ alles zusammen eine solide Ausstattung für einen gewichtigen Akteur mit Einfluss, welcher offenbar weit über die Gebietsebene hinausging. Für Kasymovas herausgehobene Position spricht auch, dass der Kolchos später nach ihr benannt wurde und dass die nachmalige Siedlung an seiner Stelle bis heute ihren Namen trägt.

Ihr Fall stellt mit großer Wahrscheinlichkeit keine Ausnahme dar. Tommaso Trevisani und Sergei Abashin schreiben ebenfalls über die machtvolle Stellung des Kolchosvorsitzenden (rais) und über die Bedeutung von horizontalen wie vertikalen Netzwerken, in welche diese Vorsitzenden eingebettet waren. Wichtig war auch die Pflege und Aufrechterhaltung dieser Netzwerke - um die eigene Stellung der Vorsitzenden zu sichern, aber auch, um die ihnen unterstehenden Großbetriebe im sowjetischen Wirtschaftssystem erfolgreich zu leiten. ${ }^{305}$

Für unseren Fall von Samarkand und den Streit um die knappe Ressource Land bedeutete die Funktionalität der Netzwerke, dass die Kolchosleitungen in

303 Das Video befindet sich im Archiv British Pathé und kann unter folgender Adresse abgerufen werden: https://www.youtube.com/watch?v=5zoXCDYvQm4 (letzter Zugriff am 30.06.2020). 304 Im Zweiten Weltkrieg wurden Kinder aus dem europäischen Teil der UdSSR evakuiert, die ihre Eltern verloren hatten; sie wurden von den lokalen Familien z.B. in Usbekistan adoptiert. Besonders bekannt, auch durch eine Verfilmung, wurde die Geschichte des Taschkenter Paars Bahri Akramova und Shoahmad Shomahmudov.

305 Trevisani 2010, S. 72. Abashin 2015, S. 357-358. 
Konflikten nicht nur den ressortgebundenen Schutz des Landwirtschaftsministeriums genossen, sondern höchstwahrscheinlich auch persönliche Fürsprecher auf unterschiedlichen Entscheidungsebenen hatten. Die Kolchosvorsitzenden konnten damit sowohl die wirtschaftlichen Interessen der Kolchosen verteidigen wie auch ihre eigenen befördern. Sie waren wichtige eigenständige Akteure, die zudem über Landressourcen verfügten und damit eine starke Position innehatten. Es mag provokativ klingen, aber offensichtlich konnten sie die Nachfrage nach Bauland in der Bevölkerung viel effektiver befriedigen als die städtischen Behörden. An dem Beispiel wird auch deutlich, wie stark die Grenzen zwischen staatlichen und privaten Akteuren verschwimmen; sichtbar wird, welche verschiedenen Einflusspositionen eine Person zugleich innehaben konnte und welche Folgen das für eine zentral gesteuerte Planung mit sich brachte.

Ein ähnliches Konfliktschema wie anhand der Engels- und Kaganovich-Kolchosen beschrieben kam im Zuge neuer Bauprojekte immer wieder auf. Der Ball ging aber offenbar mehrmals hin und her, denn der Stadt gelang es immer wieder, größere Landabschnitte für neue Bauprojekte zu bekommen, so zum Beispiel für die Mikroraions A und B im Westen der Stadt. ${ }^{306}$ Der Flickenteppich aus unterschiedlichster Bebauung wuchs immer weiter.

In einer so nüchternen wie vernichtenden Bestandsaufnahme in einem Informationsblatt aus dem Jahr 1963/64 werden die Verstöße gegen den Generalplan erneut thematisiert: Rechtswidrig gebaute Häuser stehen im Weg von geplanten Straßen, Magistralen und unterirdischen Versorgungsleitungen, was zu Kostensteigerung bei weiteren Bauarbeiten in der Stadt führe. Der unbekannte Autor kritisiert außerdem, dass die „schändliche Praxis“ hätte verhindert werden können, wenn ,die Leiter der lokalen Partei- und der Sowjetorgane die Ziele und Aufgaben des Städtebaus besser verstünden“ “. ${ }^{307}$ Der Vorwurf deutet darauf hin, dass die entsprechenden Sowjetorgane in dem Konflikt Partei für die Kolchosen ergriffen hätten.

Die spätere Gesetzgebung bekräftigt die Vermutung. Die Interessen der Kolchosen wurden Mitte bis Ende der 1960er-Jahre tatsächlich gestärkt - zum Beispiel durch eine Verordnung des Zentralkomitees der KPdSU vom 16. Juli 1966. Diese kritisierte die Verschwendung von wertvollem Kolchosland durch unberechtigte großflächige Bauvorhaben und verpflichtete die Städte und Planungsorganisationen, ihre Bebauungspläne mit den Kolchosen abzustimmen. ${ }^{308} \mathrm{Im}$

306 SamOGA, f. 1617, op. 1, d. 252, 11. 63-64

307 SamOGA, f. 1617, op. 1, d. 1, 1. 53-52.

308 SamOGA, f. 1617, op.1, d. 210, 1. 88-89. 
Jahr 1968 wurde ein großes Gesetzesprojekt für die „Grundlagen der Landnutzung“ auf den Weg gebracht und in der Presse diskutiert. ${ }^{309}$ Im Dezember des selben Jahres wurde das Projekt verabschiedet und es stellt damit das erste Regelwerk dieser Art in der Geschichte der UdSSR dar. ${ }^{310}$ Unter anderem wird darin die Entnahme von fruchtbaren, bewässerten und bestellten Böden zum Zwecke einer nicht-landwirtschaftlichen Nutzung nur in äußersten Ausnahmefällen erlaubt, und dies nur bei ausdrücklicher Zustimmung der Kolchosversammlung sowie bei gleichzeitigem Vorliegen einer Genehmigung seitens des Ministerrates der jeweiligen Republik. ${ }^{311}$

Ich konzentriere mich hier vor allem auf die Vorgänge der 1950er- und 60er-Jahre, doch ein flüchtiger Blick in die Akten der 70er-Jahre zeigt, dass die Konflikte nicht aufhörten. Auch im Jahr 1974 beklagte der Leiter der Architektur- und Planungsbehörde Sadykov, dass mehrere Kolchosen Land nicht nur an private Menschen, sondern auch an Organisationen und Institutionen unberechtigterweise und ohne Beachtung des existierenden Bebauungsplans übergeben hätten. ${ }^{312}$ Samarkand wuchs weiter und Bedarf an Bauland herrschte nicht nur bei einfachen Bürgern. Offensichtlich war es selbst für staatliche Akteure zum Teil leichter, an Bauland für ihre Vorhaben über die Kolchosleitungen zu gelangen als auf dem offiziellen Weg.

Um die Mechanismen dieser Prozesse und ihre Bedeutung für die Stadt genau nachvollziehen zu können, bedürfte es weiterer Forschung, doch auch das hier gesammelte Material liefert schon ausreichend Anhaltspunkte für eine Erklärung der Gesamtsituation: Territorien, die an die Stadt überschrieben werden sollten, hatten für die Kolchosen zukünftig keinen landwirtschaftlichen Nutzen mehr. Durch ihre gesetzliche Position sowie über die persönliche Stellung einzelner Vorsitzender hielten sie allerdings noch eine Zeitlang nach Bekanntwerden der entsprechenden Pläne die Kontrolle über die betreffenden Ländereien. Aufgrund von Knappheit und einer hohen Nachfrage verwandelte sich Grund und Boden in eine „Ware“, die, mutmaßlich nicht ohne Gegenleistungen, an Interessenten „verteilt“ werden konnte. Für eine ständige Nachfrage sorgte die schnell wachsende Bevölkerung ebenso wie die voranschreitende Industrialisierung, während der staatliche Wohnungsbau den wachsenden Bedarf nicht decken konnte. Größere und kleinere Konflikte waren hiermit vorprogrammiert.

309 Kuznetsov 1968, S. 7.

310 Gesetz der UdSSR Nr. 3401-VII vom 13.12.1968 „Über die Grundlagen der Landnutzung“

311 Gesetz der UdSSR Nr. 3401-VII vom 13.12.1968, Artikel 16.

312 SamOGA, f. 1658, op. 1, d. 477, 1. 1-4. 